\title{
Model-Based Decision Support in Manufacturing and Service Networks
}

\section{Andreas Fink, Natalia Kliewer, Dirk Mattfeld, Lars Mönch, Franz Rothlauf, Guido Schryen, Leena Suhl \& Stefan Voß}

\section{Business \& Information Systems Engineering \\ The International Journal of WIRTSCHAFTSINFORMATIK \\ e-ISSN 1867-0202 \\ Bus Inf Syst Eng \\ DOI 10.1007/s12599-013-0310-4}

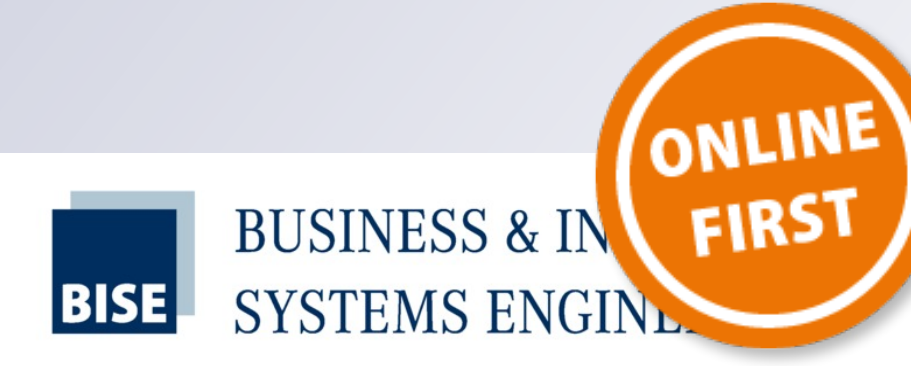

$6 / 2013$

Music as a Service as an Alternative to Music Piracy?

Jonathan Dörr, Thomas Wagner, Alexander Benlian, Thomas Hess

Low-Effort Recommendation System with High Accuracy Jella Pfeiffer, Michael Scholz

Digital Natives and Digital Immigrants Qian (Emily) Wang, Michael D. Myers, David Sundaram

Mergers and Acquisitions in the Software Industry Markus Schief, Peter Buxmann, Dirk Schiereck

Process-Driven Data Quality Management

Paul Glowalla, Ali Sunyaev

Springer Gabler 
Your article is protected by copyright and all rights are held exclusively by Springer Fachmedien Wiesbaden. This e-offprint is for personal use only and shall not be selfarchived in electronic repositories. If you wish to self-archive your article, please use the accepted manuscript version for posting on your own website. You may further deposit the accepted manuscript version in any repository, provided it is only made publicly available 12 months after official publication or later and provided acknowledgement is given to the original source of publication and a link is inserted to the published article on Springer's website. The link must be accompanied by the following text: "The final publication is available at link.springer.com". 


\title{
Model-Based Decision Support in Manufacturing and Service Networks
}

\author{
Considering integration issues taking into account the autonomy of the decision-making \\ entities in face of information asymmetry, the modeling of preferences of the \\ decision-makers, efficiently determining robust solutions and a reduction of the time \\ needed for model building and usage are challenges that should be considered in future \\ research efforts for model-based decision support in manufacturing and service networks. \\ The paper proposes a research agenda that requires an interdisciplinary collaboration of \\ business and information systems engineering researchers with scientists from \\ management science, computer science, and operations research. Some illustrative \\ examples of relevant research results are presented.
}

DOI 10.1007/s12599-013-0310-4

\section{The Authors \\ Prof. Dr. Andreas Fink \\ Helmut-Schmidt-Universität \\ Hamburg \\ Hamburg \\ Germany}

Prof. Dr. Natalia Kliewer

Freie Universität Berlin

Berlin

Germany

Prof. Dr. Dirk Mattfeld

Technische Universität Braunschweig Braunschweig

Germany

Prof. Dr. Lars Mönch ( $\varangle)$

FernUniversität in Hagen

58097 Hagen

Germany

Lars.Moench@fernuni-hagen.de

Prof. Dr. Franz Rothlauf

Johannes Gutenberg-Universität

Mainz

Mainz

Germany

Prof. Dr. Guido Schryen

Universität Regensburg

Regensburg

Germany

Prof. Dr. Leena Suhl

Universität Paderborn

Paderborn

Germany
Prof. Dr. Stefan Voß

Universität Hamburg

Hamburg

Germany

Received: 2013-03-10

Accepted: 2013-09-22

Accepted after two revisions by the editors of the special focus.

This article is also available in German in print and via http://www. wirtschaftsinformatik.de: Fink $A$, Kliewer N, Mattfeld D, Mönch L, Rothlauf F, Schryen G, Suhl L, Voß S (2014) Modellbasierte Entscheidungsunterstützung in Produktions- und Dienstleistungsnetzwerken. WIRTSCHAFTSINFORMATIK. doi: 10.1007/ s11576-013-0402-2.

(C) Springer Fachmedien Wiesbaden 2014

\section{Relevance and Timeliness of the Topic for Business and Information Systems}

The sheer size of today's highly interdependent, global supply chains, as well as the rapid pace of change and the presence of different types of uncertainties make decision support in manufacturing and service networks challenging. The interaction of the various entities in manufacturing or service networks that consume capacity, such as parts or transportation orders, is not yet well understood. It can be observed that automation on the physical level has become increasingly important, which is due to the need to increase productivity, reduce costs, and improve the quality of the manufacturing and service process. At the same time, this type of automation reduces the ability to use human beings to make critical decisions in certain situations because of an increasing decision complexity and the necessity to react at very short notice in a progressively dynamic environment. Decision models can mitigate these disadvantages and support planning and control decisions.

There is evidence that model-based decision support often results in decisions that lead to better customer service, more asset utilization, and reduced inventory. Hence, there is a strong economic need to extend and improve the model-based decision support in future manufacturing and service networks.

In this paper, we focus on the use of model-based decision support, also known as business analytics, rather than on data-driven decision support and business intelligence, to tackle these challenges. The importance of application systems that enable model-based decision support is demonstrated, for instance, by the success story of Kiva Systems, a company recently acquired by Amazon for $\$ 775$ million US dollars, specializing in the use of mobile robots in distribution facilities (cf. D'Andrea 2012). Kiva Systems has designed and implemented a movement infrastructure based on a multi-agent architecture that 
allows robots to carry loads in small areas without colliding. Model-based adaption and learning algorithms have been proposed, which allow performance of the robots to be improved over time.

Nowadays, application systems allow for the use of large amounts of structured digital data for decision-making. In recent years the performance of hard- and software components has been dramatically improved, distributed systems can be implemented quite easily on the basis of modern middleware. Also, information technology services are widely available through cloud-based solutions. Powerful algorithms that combine heuristic and exact approaches in a sophisticated way are routinely developed (cf. Maniezzo et al. 2009). Hence, in contrast to the situation two decades ago, large real-world models can be solved within a reasonable amount of time. A representative example is the use of decomposition techniques, linear programming, and heuristics to plan IBM's enterprise semiconductor supply chain (cf. Degbotse et al. 2013). The result is a unified production, shipping, and distribution plan that improved the on-time delivery performance by $15 \%$ and reduced inventory by $25-30 \%$. The integrated production planning and scheduling in Ford plants (cf. Barlatt et al. 2012) is another recent example that demonstrates large improvements in workplace planning and production scheduling. The proposed approach reveals significant reductions in overtime wages, inventory costs, and premium freight charges.

As another example, large-scale vehicle routing problems at Coca-Cola involving 10,000 trucks per day were resolved (cf. Kant et al. 2008). The result was an annual delivery cost saving of $\$ 45$ million based on the 1.5 billion cases where the dispatches were planned using the new software. In addition, there were major improvements in customer service.

It is expected that recent technological trends, for example, in-memory data management (cf. Plattner and Zeier 2011) will lead to extended and modified application scenarios. In spite of these favorable conditions, the broad application of modern approaches and corresponding application systems for decisionmaking in real-world settings remains limited. The functionality provided by current application systems, especially by packaged software systems, does not take advantage of these new, favorable conditions for decision-making. This situa- tion requires that advanced decision support systems have to be designed, that are based on improved algorithms and their interplay with information technologies. We expect that tackling the following challenges will contribute significantly to achieving broad application of efficient decision support solutions suitable for real-world use:

Challenge 1: Integration is important because decisions made in manufacturing and service networks are distributed in space and time and involve different decision-makers that have their own individual goals. Therefore, we have to research how the integration of different subsystems and the related decisions can be ensured in manufacturing and service networks through, for instance, negotiation or planning activities. In this context, it is also important to examine how preferences of human decisionmakers can be modeled in application systems, as this is an important step towards automated decision-making.

Challenge 2: Aspects of robustness are important in order to take into account uncertainty. One important research question is how it is possible to make decisions that can deal with the dynamic and stochastic behavior of the underlying manufacturing and service network.

Challenge 3: Modeling is still difficult and time-consuming. Therefore, the cycle of model building and usage has to be simplified to increase the effectiveness of the intended model-based decision support. Appropriate human-machine interfaces have to be considered, as people are the most flexible resource.

The remainder of this paper is organized as follows. In Sect. 2, we describe the challenges to be addressed in more detail. Scientific methods relevant to solving these problems are discussed in Sect. 3. Relevant academic disciplines and possible interdisciplinary cooperation required is outlined in Sect. 4. Examples for initial research results are presented in Sect. 5.

\section{Problem Description and Research Challenges}

\subsection{Overall Setting}

The information system of a manufacturing and service network can consist of several subsystems. On the planning and control level, each of the subsystems contains an internal model that represents the data of the corresponding subsystem of the physical network, for instance, all the machines and jobs of a single wafer fabrication facility that is part of an enterprise belonging to the semiconductor supply chain. Each of the planning and control subsystems, for example, Enterprise Resource Planning (ERP) systems or Manufacturing Execution Systems (MESs), can also include several decision models that are based on data of the internal models and provide instructions for other planning and control subsystems or the objects in the physical network. These decision models form the kernel of any decision support system for complex manufacturing and service networks.

The design of appropriate decision support functionality represented by the corresponding decision models is important for our research agenda. When identifying specific research problems, we have to take into account the following insights that put the ideal decisionmaking setting from the previous paragraph into perspective:

1. The internal models often contain data that is not appropriate for the required decisions, because the data is too coarse-grained, the current state is not properly reflected by the data, or historical data, needed to estimate parameters of stochastic models, is missing. When designing a decision model, it is important to clarify how to estimate and maintain the required data in a rapidly changing environment typical within manufacturing and service networks.

2. The planning and control subsystems are not fully automated, i.e., human decision-makers are involved that can make their own decisions or overrule decisions made by application systems. Therefore, it is important to know, who the recipient of a decision is, i.e., a human being or a machine. In the latter case, default actions have to be taken into account when the situation arises that implicit assumptions, for instance, with respect to the availability of jobs or resources, are violated. This allows for a higher degree of automation in decision-making that accompanies the increasing level of automation in the physical networks.

We assume that modern hard- and software infrastructure is available and allows for an effective connection of the physical 
and the information technology-related world. In principle, this permits autonomous, self-organizing, sensor-driven manufacturing and service networks. However, the design of the planning and control subsystems that take advantage of this infrastructure, for instance with respect to autonomy and the large amount of available data, is rarely discussed in the literature. In our opinion, the research challenges described in the remainder of this section are central in addressing these challenges.

\subsection{Integration Taking into Account Autonomy and Information Asymmetry in Large-Scale Networks}

This challenge is motivated by the distributed decision-making that is typical for Supply Chain Management (SCM). SCM can be seen as the coordination of a set of logistics processes that are locally controlled by several autonomous decision-making units. Coordination issues arise because there is asymmetry with respect to the point of time where the decisions are made, i.e., the involved systems operate using different time scales, with regards to the decision rights and concerning the information status of the decision-making units. The different subsystems of the overall planning and control system of the network often support local objectives that are sometimes in conflict with the overall objectives of the network. While some recent work considers decentralized decision mechanisms, e.g., negotiation procedures as described by Dudek (2009), many approaches in SCM and most of the packaged software for SCM are still based on the assumption of a single, central decision-making unit (cf. Schneeweiss 2003).

As a consequence, the design of planning and control systems that ensure the autonomy of local decision-making units has to be researched. At the same time, these units need to take into account more global decisions of units that are based on different and possibly better information. This leads to the question how planning and control should be connected in large-scale manufacturing and service networks. Coordination mechanisms for autonomous actors in distributed planning and control scenarios that consider appropriate incentives have to be analyzed.

As already discussed in the second insight in Sect. 2.1, there is a natural tradeoff between automation and autonomous decision-making, in the sense that an increasing automation, to a certain extent, restricts the autonomy of decisionmakers. Modern computing paradigms like software agents try to ensure the autonomy of decision-making entities. Such approaches however require knowledge of the explicit or implicit objectives of the decision-makers. Thus, it is also important to set appropriate incentives within coordination mechanisms that are in line with the global goals of the network. The corresponding preferences of the individual decision-makers have to be determined and eventually aggregated for group-based decision-making, as there is mostly not only a single decision-maker in manufacturing and service networks. An explicit representation of the preferences is desirable because otherwise merely implicit attitudes of individuals are represented that do not allow for automated decisions. The transformation of implicit to explicit preferences is called preference elicitation. Such methods have to take uncertain preferences into account that are typical for manufacturing and service networks which are dynamic and evolving. The uncertain preferences of a human decision-maker are characterized by imprecise, vague, and subjective utility values (cf. Lang et al. 2012). Methods from stochastic modeling and statistics are not appropriate to model this type of uncertainty in automated negotiation settings because sampling is often not possible. Many of these tools provide solutions for a steady state, with little insight into what to do in the transient setting. Therefore, alternative quantitative modeling approaches for uncertainty have to be considered.

Recently, solution approaches based on integrated planning activities have been proposed for different classes of problems in manufacturing and service networks. Typical examples are the integrated scheduling of production jobs and decision-making for automated material handling and integrated scheduling and process control. New trends in hardware and software, like in-memory data management, strongly support this development. This leads to centralized solution approaches that are in conflict with the pervasive efforts to allow more local (decentralized) decisions in networks that then have to be coordinated in an appropriate way (cf. Schütte 2012, p. 212). The question, under what conditions integrated planning approaches are reasonable or even beneficial, needs considerable further study.

\subsection{Determining Robust Solutions Considering Incomplete Information as well as Dynamic and Stochastic System Behavior}

Due to improved capabilities of the information technology, for instance, state of the art telematics systems in vehicle routing (cf. Crainic et al. 2009), it is possible nowadays to detect deviations from the situation, on which planning or control decisions were based. Typical examples are the longer travel durations, caused by traffic jams in real-time vehicle routing, demand uncertainty and uncertain supply from capacitated manufacturing systems, including Bullwhip effects, reputational risks, and quality fluctuations. The information available is often used only to a small extent in order to dynamically react to changes. We expect that the combination of improved data availability and algorithmic advances will lead to new types of decision support systems. In contrast to a pure reaction on disturbances, robustness with respect to solution quality and to the solution itself, i.e. stability, can be taken into consideration by anticipating uncertain system behavior and taking into account this anticipation during planning.

The research question is how one should design algorithms and systems that can deal with the dynamic and stochastic behavior of the underlying base system. The stability and robustness features of solution approaches are also of interest. Algorithms have to be designed that consider stochastic changes by design since the inherent uncertainty found in many real-world planning situations is currently tackled by quite simplistic methods (cf. Graves 2010). It is also challenging to investigate in which situation interventions by human decision-makers are required in automated planning and control systems.

\subsection{Reduction of the Model Building and Usage Cycle}

Modeling and simulation are established methods for decision support. At the same time, we can state that building discrete-event simulation models is still very time-consuming despite improved availability of the necessary data and the huge efforts put into automated model generation (cf. Fischbein and Yellig 2011). This limits the broad application of discrete-event simulation in industry, for example, with respect to the 
long-standing vision of supporting realtime control using simulation models based on current data from the network. As in this situation, highly detailed simulation models are required. Providing such detailed models, that include aspects from advanced automation like automated material handling systems, assembly robots, or cluster tools, i.e. specific, multi-resource mini fabs in the semiconductor manufacturing, is still far from being a trivial matter.

Simulation can be used, if fast enough and if the models are easy to build, to represent the anticipated stochastic behavior of large-scale networks in analytic solution approaches. We are capable of simulating single nodes of such networks. However, only preliminary work, especially with respect to the integration of optimization approaches, exists, addressing how to model the supply chain of a large company, for example, a global semiconductor manufacturer with dozens of wafer fabs, each containing hundreds of machines and lots, using one or several simulation models. The Supply Chain Optimization and Protocol Environment (SCOPE) (cf. Orcun et al. 2007) is an interesting example of an SCM modeling tool. However, only small-size application scenarios have been assessed and the representation of dynamic and stochastic behavior remains challenging. It is necessary to research reduced simulation models that are simple but can capture essential aspects like the dynamics of networks and load-dependent cycle times. Note that this problem is mainly caused by missing methodology and not due to inappropriate simulation tools. Up to now, there exist only a few reduction approaches that deal with a detailed modeling of process steps with regards to bottlenecks. Within these approaches, the remaining process steps are replaced by fixed time delays instead of a detailed modeling of non-bottleneck machines. As bottlenecks may change over time and the delays are load-dependent, this approach needs more attention as shown by Rose (2007), in the context of simulating a wafer fab. Such reduced simulation models would allow for the application of simulation-based optimization for largescale networks. In addition, approaches that iterate between analytic approaches, like linear programming and simulation, could be used for large-scale networks.

Appropriate situation-based parameterization of planning and control heuristics is an important problem for users and vendors of the corresponding software solutions. Moreover, preparing and analyzing the computational results is a bottleneck that often hinders the application of quantitative approaches. These questions can be treated simultaneously if appropriate knowledge-based systems are established, guiding the user to apply a certain method in the sense of a knowledge base of methods, i.e. access systems, output analyzers, and interpreters that support the analysis of the results afterwards. While such tools have been proposed for specific methods like linear programming (cf. Greenberg 1996) or discrete-event simulation (Belz and Mertens 1994), more research is clearly needed for decision support systems that are based on different methods. Moreover, assistance systems have to be developed that support the user of a certain approach, for example, when appropriate parameters have to be set.

Large computing times are still a barrier for applying quantitative methods, for example, when the approaches are intended for interactive what-if-scenarios. For instance, Degbotse et al. (2013) report that single planning instances based on enterprise data can be solved in a few hours, while multiple data sets require up to one day. Therefore, it has to be researched, which specific decisionmaking problems in manufacturing and service networks are suitable for parallel computing. Potential candidates are large-scale stochastic production planning problems or scheduling problems.

\section{Scientific Methods Relevant to Solving the Problems}

We discuss methods that can be useful to tackle instances of the three classes of challenges presented in Sects. 1 and 2 in the first three subsections. Since performance assessment issues are important for all the challenges, we discuss these in Sect. 3.4.

\subsection{Integration Problems}

It has to be investigated whether the distributed decision-making paradigm proposed by Schneeweiss (2003), or other basic organizational forms, establish a framework that can be refined by an appropriate combination of centralized and decentralized solution approaches. Little is known of how to anticipate the relevant characteristics of other involved decision-making units. Multiagent-systems (MAS) are a software paradigm that is useful in implementing distributed decision-making approaches. However, the number of successful implementations is limited. A careful comparison of the integrated (centralized) approaches with the corresponding decentralized approaches, with respect to solution quality and computing time, is necessary. Finally, incentives for autonomous actors can be determined using mechanisms from game theory. In addition, rules for selecting the outcome leading to appropriate results even in settings where agents might not disclose their preferences can also be determined by mechanism design.

Modeling approaches for uncertainty, with respect to preference elicitation, can be based on Fuzzy set theory or alternative uncertainty theories. Agent-based simulation can be applied to evaluate the modeling of preferences.

\subsection{Problems Related to Computing Robust Solutions}

Typical research efforts in the robustness domain deal with the design and assessment of online algorithms or with appropriate rescheduling algorithms. We expect that methods from stochastic programming and approximate dynamic programming, i.e., Markov decision processes, can be used to design robust algorithms for a large class of problems.

In its simplest form, a stochastic program is based on the idea that a decision is made in the first stage. Then some random events occur that affect the outcome of the first-stage decision. A recourse decision can be made in the second stage to compensate for any undesirable effects that might have been experienced as a result of the decision made in the first stage. The first-stage decision is made in such a way that the future effects are taken into account by considering the recourse function that is the expected value of making this decision.

The main idea of Markov decision processes consists of determining a policy that specifies the next action that a decision-maker chooses when a certain state is reached. The policy is determined in such a way that the expected reward is maximized.

Simulation can be applied in sampling methods, i.e., the objectives are assessed using simulation. The same ap- 
proach is taken in simulation-based optimization approaches, where metaheuristics are combined with simulation to assess the objective function in the presence of uncertainty.

\subsection{Problems Concerning the Reduction of the Model Building and Usage Cycle}

In order to make the model building process easier, the reduced simulation model approach for single nodes of a supply chain has to be extended to entire supply chains. Since it seems impossible, and unnecessary, to represent all the nodes for a large-size manufacturing or service network in a single discrete-event simulation model, methods from distributed simulation have to be applied when the detailed models are compared with the reduced approaches. We believe that combinations of detailed or reduced discreteevent simulation models with metamodels, for example, from multivariate regression, deterministic flow line models, or system dynamics, are promising. Only initial results are available for the applicability of non-linear fluid flow systems, modeled by partial differential equations, to describe the expected behavior of supply chains.

Techniques used in expert systems are potential candidates that can aid in the design of access systems, output analyzers, and interpreters. Methods from machine learning have to be applied within assistance systems to allow for a situation-dependent selection of parameters for heuristics.

To deal with the large computational burden typical for solving decision problems in manufacturing and service networks, decomposition approaches are of interest. Metaheuristics and the combination of these with mathematical programming approaches, i.e. matheuristics, where the mathematical programming approaches deal with the highly constrained subproblems, can be used to obtain high-quality solutions. In addition, the subproblems can be solved in parallel. Since powerful parallel computers, both traditional multi-core and cluster systems and more specialized architectures like the Compute Unified Device Architecture (CUDA), have recently become relatively cheap it is possible to use such resources in companies. This includes self-operating such systems or accessing the resources in the cloud. Only initial results are available for solving scheduling problems or vehicle routing problems based on metaheuristics using CUDA (cf., for example, Schulz et al. 2013).

\subsection{Performance Assessment Issues}

The performance of the proposed algorithms can either be assessed by using existing bench-mark instances or new, randomly generated problem instances. However, the simulation-based performance assessment of new planning and control approaches before the deployment in a real-world setting is highly desirable in such a dynamic and stochastic environment, as controlled experiments in the industrial context of manufacturing and service networks seem to be almost impossible.

The novel approaches have to be integrated prototypically into existing application systems. Field tests with these prototypes are highly desirable so as to assess the performance of the proposed approaches in settings that are different from those in research labs. The challenges involved in developing larger software systems and field tests are the long development cycles at universities caused by small research teams, the lacking robustness of the prototypes, and organizational problems in the companies due to a missing alignment between business processes and planning models, technical integration problems, a lack of commitment of the pilot users in the companies, and the fact that academic research labs are often unable to react quickly and effectively to solve problems with the software. Based on the feedback from the field tests, the algorithms have to be improved. We strive for a generalization of the algorithms and prototypes in the sense that they should be applicable in a broad range of situations.

\section{Relevant Academic Disciplines and Interdisciplinary Cooperation Required}

The challenges discussed in Sect. 2 require a close collaboration of business and information systems engineering with other academic disciplines. Management science is responsible for identifying and analyzing business problems. This discipline is important for all three challenges since business problems are our research domain. Methods from operations research, statistics, theoretical computer science, algorithm engineering, artificial intelligence, and machine learning have to be used to analyze the problems and to design appropriate algorithms for the three challenges. Software and data engineering methods have to be applied to build software prototypes.

In addition, we need to consider economic problems and mechanisms from a more algorithmic point of view for Challenge 1, i.e. a computer science-based view, within the framework of the interdisciplinary area of computational mechanism design (cf. Nisan et al. 2007). Findings from psychology and the behavioral sciences can be considered with respect to possible incentives of semiautomated planning and control mechanisms within Challenge 1 and the design of human-computer interaction within Challenge 3.

Let us consider the following example belonging to the first challenge that demonstrates the need for interdisciplinary research. The integrated scheduling of production jobs and preventive maintenance activities will be considered. The algorithms to be developed should be embedded into the MES of a large company. First, we have to analyze the problem. It is especially important to choose appropriate performance measures that support the global business goals of the company. Methods from management science are required to work on this task. After the problem has been well described, a mixed integer program is formulated to gain first insights. This requires operations research knowledge. We need methods from theoretical computer science to study the computational complexity of the problem. Assuming that the problem is NP-hard, we would have to develop an efficient metaheuristic approach to solve large-size instances in a reasonable amount of time. This requires knowledge in artificial intelligence and algorithm design. Machine learning approaches can be useful to select the various parameters of the metaheuristic in a situation-dependent manner. Since we are interested in using the proposed method in the company-wide MES, we have to encapsulate the provided scheduling functionality within a web service that allows us to use data from the MES and to deploy the schedules in the MES. Methods heavily relying on software and data engineering are required for developing this software prototype. 
Table 1 Mapping of research challenges to initial results

\begin{tabular}{|c|c|c|}
\hline Challenge & Reference & Content \\
\hline \multirow[t]{6}{*}{ Integration in networks } & Mönch (2006) & MAS for wafer fab scheduling \\
\hline & Steinzen et al. (2010) & Integration of crew and vehicle scheduling \\
\hline & Fink and Homberger (2013) & Automated negotiation mechanism \\
\hline & Driessel and Mönch (2012) & Integrated scheduling and automated transport \\
\hline & Pfeiffer et al. (2008) & Reference point-based modeling of preferences \\
\hline & Lang et al. (2012) & Fuzzy sets to model uncertainty in preferences \\
\hline \multirow[t]{2}{*}{ Determination of robust solutions } & Ehmke et al. (2012) & Usage of real-time data in city logistics \\
\hline & Dück et al. (2012) & Robust crew and airline scheduling \\
\hline \multirow[t]{4}{*}{ Reduction of the model building and usage cycle } & Fink and Voß (2003) & Fast design/implementation of metaheuristics \\
\hline & Rothlauf (2011) & Design of metaheuristics \\
\hline & Ehm et al. (2011) & Supply chain simulation using reduced models \\
\hline & Bilyk and Mönch (2012) & Parallel computing in scheduling \\
\hline
\end{tabular}

\section{Examples of Initial Results}

We exemplify some research efforts in the remainder of this section. In Table 1, the corresponding papers are assigned to the challenges discussed in Sect. 2.

Mönch (2006) proposes a distributed, hierarchical production control system for large-scale manufacturing systems. The system is implemented as MAS. However, it would be interesting to include production planning approaches into the MAS. An integrated optimization approach for the planning steps crew scheduling and vehicle scheduling is presented by Steinzen et al. (2010). Fink and Homberger (2013) are developing an automated negotiation mechanism for project scheduling, where information asymmetry and conflicting objectives are taken into account. Driessel and Mönch (2012) propose an integrated scheduling and automated material handling approach that extends the shifting bottleneck heuristic to this situation. However, only a simplified, automated material handling system is considered. Hence, much more research is needed to incorporate real-world issues.

Multi-objective, evolutionary algorithms are proposed by Pfeiffer et al. (2008) taking into account preferences of the decision-makers in form of reference points. However, the proposed approach has to be evaluated using many additional application scenarios. Often linguistic uncertainty is associated with the preferences of the decision-makers. Lang et al. (2012) deal with the elicitation, modeling, and processing of un- certain preferences of human decisionmakers within electronic negotiations using Fuzzy sets. However, uncertainty theories different from Fuzzy sets should be tested too. Applications in the supply chain coordination domain should be considered.

Ehmke et al. (2012) show how realtime data can be used to improve decisions in the transportation logistics domain. Dück et al. (2012) propose approaches for robust crew and aircraft scheduling, taking into account the delays and disturbances typical for air transportation.

The design of metaheuristics by means of an object-oriented framework for industrial planning problems is discussed by Fink and Voß (2003). Rothlauf (2011) classifies metaheuristics and demonstrates how such heuristics can be designed using problem- and instancespecific knowledge. Ehm et al. (2011) consider the simulation of supply chains in semiconductor manufacturing. Reduced simulation techniques are applied for a small-size supply chain. However, the method should be extended to largescale supply chains. Bilyk and Mönch (2012) propose a variant of the shifting bottleneck heuristic that is performed on a parallel computer to solve large-scale scheduling problems for semiconductor wafer fabrication facilities. This allows using modern metaheuristics to solve the resulting subproblems. Designed simulation experiments for using metaheuristics are desirable.

Summarizing the insights from the discussion in this paper, we propose the following research agenda for the next several years.

Challenge 1: We will research how planning and control can be linked in large-scale manufacturing and service networks by considering specific examples from production planning and scheduling. We will extend the knowledge with respect to preference modeling for decision-makers by considering alternative uncertainty theories and by studying application examples. Coordination mechanisms for autonomous actors that take incentives into consideration will be analyzed. We are interested in improving our understanding of the advantages and limitations of integrated planning approaches compared with decentralized, coordinated approaches.

Challenge 2: We will design, implement, and assess algorithms and the corresponding application systems that are able to take into account the dynamic and stochastic behavior of the underlying base system. Initially, we will consider dynamic and stochastic counterparts of well-understood, static problems with deterministic data in transportation, production planning, and scheduling. We are interested in understanding the limitations of the rather simplistic methods that are currently used to tackle such problems. Based on the gained insights, we will work on more advanced techniques.

Challenge 3: We will design, implement, and test techniques that allow for the simulation of large-scale supply chains. Based on these simulation models, we will work on approaches for large- 
scale manufacturing and service networks that hybridize analytic approaches and simulation. We will research how we can use such simulation-based methods in commercial application systems. We will also work on the situation-based parameterization of heuristics used for decision-making support in manufacturing and service networks. We will research the application potential of parallel computing techniques by studying domain-specific examples.

\section{Conclusions}

In this paper we have discussed several challenges for model-based decision support in manufacturing and service networks. The main challenges are related to distributed decision-making in largescale manufacturing and service networks, the necessity to take dynamic and stochastic system behavior into account when decisions are made, and to improve the model building and usage cycle. We explained why we believe that tackling these challenges is important to achieve a broad application of efficient decision support solutions suitable for real-world use. It has also been demonstrated why the required efforts have to be interdisciplinary by nature. A research agenda for the next several years has been proposed.

\section{Acknowledgements}

The authors would like to thank Reha Uzsoy, North Carolina State University, for useful comments on this paper.

\section{References}

Barlatt AY, Cohn A, Gusikhin O, Fradkin Y, Davidson R, Batey J (2012) Ford motor company implements integrated planning and scheduling in a complex automotive manufacturing environment. Interfaces 42(5):478-491

Belz R, Mertens P (1994) SIMULEX - a multiattribute DSS to solve rescheduling problems. Annals of Operations Research 52(3):107-129

Bilyk A, Mönch L (2012) Variable neighborhood search-based subproblem solution procedures for a parallel shifting bottleneck heuristic for complex job shops. In: Proc of IEEE conference on automation science and engineering, Seoul, pp 415-420

Crainic TG, Gendreau M, Potvin JY (2009) Intelligent freight-transportation systems: assessment and the contribution of op- erations research. Transportation Research Part C: Emerging Technologies 16(6):541557

D'Andrea R (2012) A revolution in the warehouse: a retrospective on Kiva systems and the grand challenges ahead. IEEE Transaction on Automation Science and Engineering 9(4):638-639

Degbotse A, Denton BT, Fordyce K, Milne RJ, Orzell R, Wang C-T (2013) IBM blends heuristics and optimization to plan its semiconductor supply chain. Interfaces 43(2):130-142

Dudek G (2009) Collaborative planning in supply chains, 2nd edn. Springer, Heidelberg

Driessel R, Mönch L (2012) An integrated scheduling and material handling approach for complex job shops: a computational study. International Journal of Production Research 50(20):5966-5985

Dück V, lonescu L, Kliewer N, Suhl L (2012) Increasing stability of crew and aircraft schedules. Transportation Research Part C: Emerging Technologies 20(1):47-61

Ehm $\mathrm{H}$, Wenke $\mathrm{H}$, Mönch L, Ponsignon $\mathrm{T}$, Forstner $L$ (2011) Towards a supply chain simulation reference model for the semiconductor industry. In: Proc of the 2011 winter simulation conference, Phoenix, USA, pp 2124-2135

Ehmke J, Meisel S, Mattfeld DC (2012) Floating car based travel times for city logistics. Transportation Research Part C: Emerging Technologies 21(1):338-352

Fink A, Voß S (2003) Anwendung von Metaheuristiken zur Lösung betrieblicher Planungsprobleme - Potenziale und Grenzen einer softwaretechnischen Unterstützung WIRTSCHAFTSINFORMATIK 45(4):395-407

Fink A, Homberger J (2013) An ant-based coordination mechanism for resourceconstrained project scheduling with multiple agents and cash flow objectives. Flexible Services and Manufacturing Journal 25(1/2):94-121

Fischbein SA, Yellig E (2011) Why it is so hard to build and validate discrete event simulation models of manufacturing facilities. In: Kempf K, Keskinocak P, Uzsoy R (eds) Planning production and inventories in the extended enterprise: a state of the art handbook, vol 2. Springer, Heidelberg, pp 271-288

Graves SC (2010) Uncertainty and production planning. In: Kempf $\mathrm{K}$, Keskinocak $\mathrm{P}$ Uzsoy R (eds) Planning production and inventories in the extended enterprise: a state of the art handbook, vol 1. Springer, Heidelberg, pp 83-102

Greenberg HJ (1996) The ANALYZE rulebase for supporting LP analysis. Annals of Operations Research 65(1):91-126

Kant G, Jacks M, Aantjes C (2008) CocaCola enterprises optimizes vehicle routes for efficient product delivery. Interfaces 38(1):40-50

Lang F, Fink A, Schryen G (2012) Elicitating, modeling, and processing uncertain human preferences for software agents in electronic negotiations: an empirical study. In: Proc of the international conference on information systems (ICIS) 2012, Association for Information Systems

Maniezzo V, Stützle T, Voß S (eds) (2009) Matheuristics: hybridizing metaheuristics and mathematical programming. Annals of information systems, vol 10. Springer, Heidelberg

\section{Abstract \\ Andreas Fink, Natalia Kliewer, Dirk Mattfeld, Lars Mönch, Franz Rothlauf, Guido Schryen, Leena Suhl, Stefan Voß \\ Model-Based Decision Support in Manufacturing and Service Networks}

In this paper, we sketch some of the challenges that should be addressed in future research efforts for modelbased decision support in manufacturing and service networks. This includes integration issues, taking into account the autonomy of the decision-making entities in face of information asymmetry, the modeling of preferences of the decision makers, efficiently determining robust solutions, i.e. solutions that are insensitive with respect to changes in the problem data, and a reduction of the time needed for model building and usage. The problem solution cycle includes problem analysis, the design of appropriate algorithms and their performance assessment. We are interested in a prototypical integration of the proposed methods within application systems, which can be followed up with field tests of the extended application systems. We argue that the described research agenda requires the interdisciplinary collaboration of business and information systems engineering researchers with colleagues from management science, computer science, and operations research. In addition, we present some exemplifying, illustrative examples of relevant research results.

Keywords: Model-based decision support, Manufacturing and service networks, Research areas in business and information systems engineering 
Mönch L (2006) Autonome und kooperative Steuerung komplexer Produktionsprozesse mit Multi-Agenten-Systemen. WIRTSCHAFTSINFORMATIK 48(2):107-119

Nisan N, Rougarden T, Tardos E, Vazirani VV (eds) (2007) Algorithmic game theory. Cambridge University Press, New York

Orcun S, Asmundsson JM, Uzsoy R, Clement JP, Pekny JF, Rardin RL (2007) Supply chain optimization and protocol environment (SCOPE) for rapid prototyping and analysis of complex supply chains. Production Planning \& Control 18(5):388-406

Pfeiffer J, Golle U, Rothlauf F (2008) Reference point based multi-objective algorithms for group decisions. In: Proc of the genetic and evolutionary computation conference, pp 697-704

Plattner H, Zeier A (2011) In-memory data management - an inflection point for enterprise applications. Springer, Heidelberg

Rose O (2007) Improved simple simulation models for semiconductor wafer fabrication facilities. In: Proc of the 2007 winter simulation conference, pp 1708-1712

Rothlauf F (2011) Design of modern heuristics: principles and application. Springer, Heidelberg

Schneeweiss C (2003) Distributed decision making, 2nd edn. Springer, Heidelberg
Schulz C, Hasle G, Brodtkorb AR, Hagen TR (2013) GPU computing in discrete optimization. Part II. Survey focused on routing problems. EURO Journal on Transportation and Logistics 2:159-186

Schütte R (2012) In-Memory-Technologien: Überlegungen zur Begründbarkeit und zum Einsatz beim Betrieb von großen Systemen, WI-Meinung/Dialog. WIRTSCHAFTSINFORMATIK 54(4):211-213

Steinzen I, Gintner V, Suhl L, Kliewer N (2010) A time-space network approach for the integrated vehicle and crew scheduling problem with multiple depots. Transportation Science 44(3):367-382 